Research Article

\title{
The Behavior of an SVIR Epidemic Model with Stochastic Perturbation
}

\author{
Yanan Zhao ${ }^{1,2,3}$ and Daqing Jiang ${ }^{1,3}$ \\ ${ }^{1}$ School of Mathematics and Statistics, Northeast Normal University, Changchun, Jilin 130024, China \\ ${ }^{2}$ School of Science, Changchun University, Changchun, Jilin 130022, China \\ ${ }^{3}$ College of Science, China University of Petroleum (East China), Qingdao 266580, China
}

Correspondence should be addressed to Daqing Jiang; daqingjiang2010@hotmail.com

Received 18 October 2013; Revised 4 May 2014; Accepted 6 May 2014; Published 24 June 2014

Academic Editor: Cristina Pignotti

Copyright (C) 2014 Y. Zhao and D. Jiang. This is an open access article distributed under the Creative Commons Attribution License, which permits unrestricted use, distribution, and reproduction in any medium, provided the original work is properly cited.

We discuss a stochastic SIR epidemic model with vaccination. We investigate the asymptotic behavior according to the perturbation and the reproduction number $R_{0}$. We deduce the globally asymptotic stability of the disease-free equilibrium when $R_{0} \leq 1$ and the perturbation is small, which means that the disease will die out. When $R_{0}>1$, we derive that the disease will prevail, which is measured through the difference between the solution and the endemic equilibrium of the deterministic model in time average. The key to our analysis is choosing appropriate Lyapunov functions.

\section{Introduction}

Epidemiology is the study of the spread of diseases with the objective to trace factors that are responsible for or contribute to their occurrence. Mathematical models are used extensively in the study of epidemiological phenomena. Most models for the transmission of infectious diseases descend from the classical SIR model of Kermack and McKendrick established in 1927; see [1]. In recent years, many researchers have discussed the SIR model allowing vaccination, that is, the SVIR model. In the epidemiology, vaccines are extremely important and widely used in the modern day world and have been proved to be the most effective and cost-efficient method of preventing infectious diseases such as measles, polio, diphtheria, tetanus, pertussis, and tuberculosis. Routine vaccination is now provided in all developing countries against all these diseases. $\mathrm{Li}$ and $\mathrm{Ma}$ [2] discuss an SIS model with vaccination. The results in [2] show that the system always has the disease-free equilibrium $P_{0}$. If the basic reproduction number $R_{0} \leqslant 1$, then $P_{0}$ is the unique equilibrium and it is globally stable. If $R_{0}>1$, then $P_{0}$ is unstable and there is an endemic equilibrium $P^{*}$ which is globally asymptotically stable under a sufficient condition. In addition, much research has been done on SVIR models; see [3-6]. In light of these results, complete determination of the global dynamics of these models is essential for their application and further development.

In fact, epidemic models are inevitably affected by environmental white noise which is an important component in realism, because it can provide an additional degree of realism in comparison to their deterministic counterparts. Recent advances in stochastic differential equations enable us to introduce stochasticity into the model of biological phenomena, whether it is a random noise in the system of differential equations or environmental fluctuations in parameters. Modeling population dynamics in random environments is a way of studying the fluctuations of population size that has been affected by the stochasticity of external factors. Recently several authors have studied stochastic biological systems; see [7-9]. In addition, some stochastic epidemic models have been studied by many authors; see [10-15]. Parameter perturbations on the transmission rate are considered in $[10,12,14,16]$. Tornatore et al. [12] study the stability of disease-free equilibrium of a stochastic SIR model with or without distributed time delay, and the same discussion is extended to a SIRS model by $\mathrm{Lu}$ [10]. To our knowledge, due to the complexity of stochastic SVIR models, this is the first time that a stability analysis for such 
system with white noise stochastic perturbations around the transmission rate $\beta$ is performed.

In this paper, we will discuss the stochastic SVIR model as follows:

$$
\begin{gathered}
d S(t)=(\Lambda-\beta S(t) I(t)-(\mu+p) S(t)+\varepsilon V(t)) d t \\
-\sigma S(t) I(t) d B(t), \\
d I(t)=(\beta S(t) I(t)-(\mu+\gamma+\alpha) I(t)) d t \\
\quad+\sigma S(t) I(t) d B(t), \\
d V(t)=(p S(t)-(\mu+\varepsilon) V(t)) d t \\
d R(t)=(\gamma I(t)-\mu R(t)) d t .
\end{gathered}
$$

SVIR models are formulated by dividing the population size $N(t)$ into four distinct groups, $S(t), V(t), I(t)$, and $R(t)$, where $S(t)$ is the numbers of a population susceptible to the disease, $I(t)$ is the number of infective members, $V(t)$ is the number of vaccinated members, and $R(t)$ is the number of who have been removed from the possibility of infection through full immunity. The parameters in the model are summarized in the following list:

$\Lambda$ : a constant input of new members into the population per unit time;

$\beta$ : transmission coefficient between compartments $S$ and $I$;

$\mu$ : natural death rate of $S, I$, and $V$ compartments;

$p$ : the proportional coefficient of vaccinated individuals for the susceptible;

$\gamma$ : recovery rate of infectious individuals;

$\varepsilon$ : the rate of losing their immunity for vaccinated individuals;

$\alpha$ : disease-caused death rate of infectious individuals.

All parameter values are assumed to be nonnegative and $\mu, \Lambda>0$. Here $B(t)$ is standard Brownian motions with $B(0)=$ 0 and with intensity of white noise $\sigma^{2}>0$. Since the last equation is independent of other equations, we investigated the following equivalent system:

$$
\begin{gathered}
d S(t)=(\Lambda-\beta S(t) I(t)-(\mu+p) S(t)+\varepsilon V(t)) d t \\
-\sigma S(t) I(t) d B(t) \\
d I(t)=(\beta S(t) I(t)-(\mu+\gamma+\alpha) I(t)) d t \\
\quad+\sigma S(t) I(t) d B(t) \\
d V(t)=(p S(t)-(\mu+\varepsilon) V(t)) d t .
\end{gathered}
$$

Model (2) always has a disease-free equilibrium $E_{0}=$ $\left(S_{0}, I_{0}, V_{0}\right)=(\Lambda(\mu+\varepsilon) / \mu(\mu+\varepsilon+p), 0, \Lambda p / \mu(\mu+\varepsilon+p))$. Define a basic reproduction number $R_{0}=\Lambda \beta(\mu+\varepsilon) / \mu(\mu+$ $\gamma+\alpha)(\mu+\varepsilon+p)$, which means the average new infections produced by one infected individual during his lifespan when the population is at $E_{0}$. By (2), a positive equilibrium
$E^{*}=\left(S^{*}, I^{*}, V^{*}\right)=((\mu+\gamma+\alpha) / \beta, \Lambda /(\mu+\gamma+\alpha)(1-$ $\left.\left.1 / R_{0}\right), p(\mu+\gamma+\alpha) / \beta(\mu+\varepsilon)\right)$ satisfies $\Lambda-\beta S^{*} I^{*}-(\mu+p) S^{*}+\varepsilon V^{*}$, $\beta S^{*} I^{*}=(\mu+\gamma+\alpha) I^{*}$, and $p S^{*}=(\mu+\varepsilon) V^{*}$.

In this paper, unless otherwise specified, we let $\left(\Omega, \mathscr{F},\left\{\mathscr{F}_{t}\right\}_{t \geq 0}, P\right)$ be a complete probability space with a filtration $\left\{\mathscr{F}_{t}\right\}_{t \geq 0}$ satisfying the usual conditions (i.e., it is increasing and right continuous while $\mathscr{F}_{0}$ contains all P-null sets). Let $B(t)$ be the Brownian motions defined on this probability space. Also let $\mathbb{R}_{+}^{n}=\left\{x \in \mathbb{R}^{n}, x_{i}>0\right.$ for all $\left.1 \leqslant i \leqslant n\right\}$.

\section{Existence and Uniqueness of the Positive Solution}

In this section, we prove that the solution of model (2) is positive and global.

Theorem 1. There is a unique solution $(S(t), I(t), V(t))$ of system (2) on $t \geqslant 0$ for any initial value $(S(0), I(0), V(0)) \in \mathbb{R}_{+}^{3}$, and the solution will remain in $\mathbb{R}_{+}^{3}$ with probability 1; namely, $(S(t), I(t), V(t)) \in \mathbb{R}_{+}^{3}$ for all $t \geqslant 0$ a.s.

Proof. Since the coefficient of the equation is locally Lipschitz continuous, for any given initial value $(S(0), I(0), V(0)) \in \mathbb{R}_{+}^{3}$ there is a unique local solution $(S(t), I(t), V(t))$ on $t \in\left[0, \tau_{e}\right)$, where $\tau_{e}$ is the explosion time [17]. To show that this solution is global, we need to show that $\tau_{e}=\infty$ a.s.

Since the following argument is similar to that of [17], we here only sketch the proof to point out the difference with it. Let $k_{0}>0$ be sufficiently large for $S(0), I(0)$, and $V(0)$ all lying within the interval $\left[1 / k_{0}, k_{0}\right]$. For each integer $k \geqslant k_{0}$, define the stopping time

$$
\begin{array}{r}
\tau_{k}=\inf \left\{t \in\left[0, \tau_{e}\right): \min \{S(t), I(t), V(t)\} \leq \frac{1}{k}\right. \text { or } \\
\max \{S(t), I(t), V(t)\} \geq k\} .
\end{array}
$$

In the sequel, we need to show $\tau_{\infty}:=\lim _{k \rightarrow \infty} \tau_{k}=\infty$ a.s.

Define a $C^{2}$-function $W: \mathbb{R}_{+}^{3^{\infty}} \rightarrow \mathbb{R}_{+}$as follows:

$$
\begin{aligned}
W(S, I, V)= & \left(s-a-a \log \frac{S}{a}\right)+(I-1-\log I) \\
& +(V-1-\log V) .
\end{aligned}
$$

Let $k \geqslant k_{0}$ and $T>0$ be arbitrary. Applying the Itô's formula, we obtain

$$
d W(S, I, V)=L W d t-\sigma(S-a) I d B(t)+\sigma(I-1) S d B(t),
$$

where $L W: \mathbb{R}_{+}^{3} \rightarrow \mathbb{R}_{+}$is defined by

$$
\begin{aligned}
L W(S, I, V)= & -\mu S-(\mu+\gamma+\alpha) I-\mu V-a \frac{\Lambda}{S}+a \beta I \\
& -a \varepsilon \frac{V}{S}-\beta S-p \frac{S}{V}+\Lambda+\frac{1}{2} a \sigma^{2} I^{2}+\frac{1}{2} \sigma^{2} S^{2} \\
& +a(\mu+p)+(\mu+\gamma+\alpha)+(\mu+\varepsilon)
\end{aligned}
$$




$$
\begin{aligned}
= & -(\mu+\beta) S+[a \beta-(\mu+\gamma+\alpha)] I-\mu V \\
& -a \frac{\Lambda}{S}-a \varepsilon \frac{V}{S}-p \frac{S}{V}+\Lambda+\frac{1}{2} a \sigma^{2} I^{2}+\frac{1}{2} \sigma^{2} S^{2} \\
& +a(\mu+p)+(\mu+\gamma+\alpha)+(\mu+\varepsilon) .
\end{aligned}
$$

Let $a=(\mu+\gamma+\alpha) / \beta$, such that $a \beta-(\mu+\gamma+\alpha)=0$; then substituting this into (6), we get

$$
\begin{aligned}
L W(S, I, V) \leq & \Lambda+\frac{1}{2}(a+1) \sigma^{2}\left(\frac{\Lambda}{S}\right)^{2}+a(\mu+p) \\
& +(\mu+\gamma+\alpha)+(\mu+\varepsilon):=K
\end{aligned}
$$

The remainder of the proof follows that in Mao et al. [17].

Remark 2. From Theorem 1 for any initial value $(S(0), I(0), V(0)) \in \mathbb{R}_{+}^{3}$, there is a unique global solution $(S(t), I(t), V(t)) \in \mathbb{R}_{+}^{3}$ a.s. of system (2). Hence

$$
\begin{gathered}
d(S+I+V) \leq[\Lambda-\mu(S+I+V)] d t \\
S(t)+I(t)+V(t) \leq \frac{\Lambda}{\mu}+e^{-\mu t}\left(S(0)+I(0)+V(0)-\frac{\Lambda}{\mu}\right) .
\end{gathered}
$$

If $S(0)+I(0)+V(0) \leq \Lambda / \mu$, then $S(t)+I(t)+V(t) \leq \Lambda / \mu$ a.s. and so the region

$$
\Gamma^{*}=\left\{(S, I, V): S>0, I>0, V>0, S+I+V \leq \frac{\Lambda}{\mu} \text { a.s. }\right\}
$$

is a positively invariant set of system (2).

\section{Asymptotic Behavior of the Disease-Free Equilibrium}

It is clear that $P_{0}=(\Lambda(\mu+\varepsilon) / \mu(\mu+\varepsilon+p), 0, \Lambda p / \mu(\mu+\varepsilon+$ $p)$ ) is a solution of system (2), which is called the diseasefree equilibrium. As mentioned in the introduction, if $P_{0}$ is globally asymptotically stable, the disease will die out after some period of time. Obviously, it is interesting to study the disease-free equilibrium for controlling infectious disease. In this section, we obtain the stability of this point mainly by stochastic Lyapunov function.

In general, consider $d$-dimensional stochastic differential equation

$$
d x(t)=f(x(t), t) d t+g(x(t), t) d B(t), \quad t \geq t_{0}
$$

with initial value $x\left(t_{0}\right)=x_{0} \in R^{d}$. Consider (10), assume $f(0, t)=0$ and $g(0, t)=0$ for all $t \geq t_{0}$. So $x(t) \equiv 0$ is a solution to (10), called the trivial solution or equilibrium position. The following theorems give conditions for the stability of the trivial solution of (10) in terms of Lyapunov function (see [18]).

Lemma 3. If there exists a positive definite decrescent radially unbounded function $V(x, t) \in C^{2,1}\left(R^{d} \times\left[t_{0}, \infty\right) ;[0, \infty)\right)$ such that $L V(x, t)$ is negative definite, then the trivial solution of (10) is stochastically asymptotically stable in the large.

Lemma 4 (strong law of large numbers). Let $M=\left\{M_{t}\right\}_{t \geq 0}$ be a real-value continuous local martingale vanishing at $t=0$. Then

$$
\lim _{t \rightarrow \infty}\langle M, M\rangle_{t}=\infty \text { a.s. } \Longrightarrow \lim _{t \rightarrow \infty} \frac{M_{t}}{\langle M, M\rangle_{t}}=0 \text { a.s. }
$$

and also

$$
\limsup _{t \rightarrow \infty} \frac{\langle M, M\rangle_{t}}{t}<\infty \text { a.s. } \Longrightarrow \lim _{t \rightarrow \infty} \frac{M_{t}}{t}=0 \text { a.s. }
$$

Based on these lemmas, now we give out the main theorems.

Theorem 5. If $R_{0} \leqslant 1$ and $\sigma^{2}<(\gamma+\alpha) \mu^{2} / \Lambda^{2}$, then the diseasefree equilibrium $P_{0}$ of system (2) is stochastically asymptotically stable in the large.

Proof. let $x=S-\Lambda(\mu+\varepsilon) / \mu(\mu+\varepsilon+p), y=I, z=V-$ $\Lambda p / \mu(\mu+\varepsilon+p)$, and $m=\Lambda / \mu(\mu+\varepsilon+p)$; then

$$
\begin{gathered}
d x(t)=[-(\mu+p) x(t)-\beta x(t) y(t)-\beta(\mu+\varepsilon) m y(t) \\
+\varepsilon z(t)] d t-\sigma[x(t)+(\mu+\varepsilon) m] y(t) d B(t), \\
d y(t)=\{\beta x(t) y(t)-[(\mu+\gamma+\alpha)-\beta(\mu+\varepsilon) m] y(t)\} d t \\
+\sigma[x(t)+(\mu+\varepsilon) m] y(t) d B(t), \\
d z(t)=[p x(t)-(\mu+\varepsilon) z(t)] d t .
\end{gathered}
$$

Define a $C^{2}$-function $W: \mathbb{R}_{+}^{3} \rightarrow \mathbb{R}_{+}$by

$$
\begin{aligned}
W(x, y, z) & =q\left(\frac{1}{2} x^{2}+c_{1} y+\frac{1}{2} c_{2} z^{2}\right)+\frac{1}{2}(x+y+z)^{2} \\
& =q W_{1}+W_{2},
\end{aligned}
$$

where

$$
\begin{gathered}
W_{1}(x, y, z)=\frac{1}{2} x^{2}+c_{1} y+\frac{1}{2} c_{2} z^{2}, \\
W_{2}(x, y, z)=\frac{1}{2}(x+y+z)^{2} .
\end{gathered}
$$

From Itô's formula, we compute

$$
\begin{aligned}
d W_{1}(x, y, z)= & L W_{1} d t+\sigma x y[x+(\mu+\varepsilon) m] d B(t) \\
& +c_{1} \sigma[x+(\mu+\varepsilon) m] y d B(t),
\end{aligned}
$$


where

$$
\begin{aligned}
L W_{1}= & x d x+\frac{1}{2}(d x)^{2}+c_{1} d y+c_{2} z d z+\frac{1}{2} c_{2}(d z)^{2} \\
= & -(\mu+p) x^{2}-\beta x^{2} y-\beta(\mu+\varepsilon) m x y+\varepsilon x z \\
& +\frac{1}{2} \sigma^{2}[x+(\mu+\varepsilon) m]^{2} y^{2}+c_{1} \beta x y \\
& -c_{1} \beta(\mu+\varepsilon) m\left(\frac{1}{R_{0}}-1\right) y+c_{2} p x z-c_{2}(\mu+\varepsilon) z^{2} \\
\leqslant & -(\mu+p) x^{2}-\left[(\mu+\varepsilon) m-c_{1}\right] \beta x y+\left(\varepsilon+c_{2} p\right) x z \\
& -c_{2}(\mu+\varepsilon) z^{2}+\frac{1}{2} \sigma^{2}\left(\frac{\Lambda}{\mu}\right) y^{2},
\end{aligned}
$$

where $R_{0} \leq 1$ is used. Here we choose $c_{1}=(\mu+\varepsilon) m$, such that $(\mu+\varepsilon) m-c_{1}=0$. Choose $c_{2}=\varepsilon / p$ and $\rho=((\mu+\varepsilon)(\mu+p)+$ $\varepsilon p) / 2 p(\mu+p)$; substituting this into (17) yields

$$
\begin{aligned}
L W_{1} \leqslant & -\left[(\mu+p)-\frac{\varepsilon}{\rho}\right] x^{2}-\left[\frac{\varepsilon}{p}(\mu+\varepsilon)-\rho \varepsilon\right] z^{2} \\
& +\frac{1}{2} \sigma^{2}\left(\frac{\Lambda}{\mu}\right) y^{2} \\
= & -\frac{\mu(\mu+\varepsilon+p)(\mu+p)}{(\mu+\varepsilon)(\mu+p)+\varepsilon p} x^{2}-\frac{\mu \varepsilon(\mu+\varepsilon+p)}{2 p(\mu+p)} z^{2} \\
& +\frac{1}{2} \sigma^{2}\left(\frac{\Lambda}{\mu}\right) y^{2} .
\end{aligned}
$$

Similarly, applying Itô’s formula to $W_{2}(x, y, z)$, we get

$$
\begin{aligned}
L W_{2}= & (x+y+z)(d x+d y+d z)+\frac{1}{2}(d x+d y+d z)^{2} \\
= & (x+y+z)[-\mu x-(\mu+\gamma+\alpha) y-\mu z] \\
= & -\mu x^{2}-(\mu+\gamma+\alpha) y^{2}-\mu z^{2}-(2 \mu+\gamma+\alpha) x y \\
& -(2 \mu+\gamma+\alpha) y z-2 \mu x z \\
\leqslant & (2 \mu+\gamma+\alpha) x^{2}-\frac{\gamma+\alpha}{2} y^{2}+(2 \mu+\gamma+\alpha) z^{2} .
\end{aligned}
$$

Therefore, we can obtain

$$
\begin{aligned}
L W= & q W_{1}+W_{2} \\
\leqslant & -\left[q \frac{\mu(\mu+\varepsilon+p)(\mu+p)}{(\mu+\varepsilon)(\mu+p)+\varepsilon p}-(2 \mu+\gamma+\alpha)\right] x^{2} \\
& -\left[\frac{\gamma+\alpha}{2}-\frac{1}{2} \sigma^{2}\left(\frac{\Lambda}{\mu}\right)^{2}\right] y^{2} \\
& -\left[q \frac{\mu \varepsilon(\mu+\varepsilon+p)}{2 p(\mu+p)}-(2 \mu+\gamma+\alpha)\right] z^{2} \\
:= & -a x^{2}-b y^{2}-c z^{2},
\end{aligned}
$$

where $q=\max \{((\mu+\varepsilon)(\mu+p)+\varepsilon p)(2 \mu+\gamma+\alpha) / \mu(\mu+\varepsilon+$ $p)(\mu+p),(2 \mu+\gamma+\alpha) 2 p(\mu+p) / \mu \varepsilon(\mu+\varepsilon+p)\}$, such that $a, c \geq 0$. When $\sigma^{2}<(\gamma+\alpha) \mu^{2} / \Lambda^{2}, L W$ is negative definite. By Lemma 3 , we conclude that, under the condition $R_{0} \leqslant 1$, the trivial solution of system (13) is stochastically asymptotically stable in the large; that is, the disease-free equilibrium $P_{0}$ of system (2) is stochastically asymptotically stable in the large.

\section{Asymptotic Behavior around the Endemic Equilibrium of the Deterministic Model}

When studying the dynamic of an epidemic system, we are interested in two problems: one is when the disease will die out which has been shown in Section 3 and the other is when the disease will prevail and persist in a population. In the deterministic models, this is solved by showing that the endemic equilibrium to the corresponding model is a global attractor or is globally asymptotically stable under some conditions. But there is no endemic equilibrium in system (2). It is plausible by studying the behavior around $E^{*}$ to reflect whether the disease will prevail. We get the following result.

Theorem 6. Let $(S(t), I(t), V(t))$ be the solution of system (2) with initial value $(S(0), I(0), V(0)) \in \mathbb{R}_{+}^{3}$. If $R_{0}>1$, then

$$
\begin{gathered}
\limsup _{t \rightarrow \infty} \frac{1}{t} \int_{0}^{t}\left\{\left[l \frac{\mu(\mu+\varepsilon+p)(\mu+p)}{(\mu+\varepsilon)(\mu+p)+\varepsilon p}-(2 \mu+\gamma+\alpha)\right]\right. \\
\quad \times\left(S(r)-S^{*}\right)^{2}+\frac{\gamma+\alpha}{2}\left(I(r)-I^{*}\right)^{2} \\
+\left[l \frac{\mu \varepsilon(\mu+\varepsilon+p)}{2 p(\mu+p)}-(2 \mu+\gamma+\alpha)\right] \\
\left.\quad \times\left(V(r)-V^{*}\right)^{2}\right\} d r \\
\leqslant \frac{1}{4} \sigma^{2}\left(\frac{\Lambda}{\mu}\right)^{2}\left(1+\left(\frac{\Lambda}{\mu}\right)^{2}\right) \text { a.s., }
\end{gathered}
$$

wherel is a positive constant defined as (29).

Proof. Define a $C^{2}$-function $W: \mathbb{R}_{+}^{3} \rightarrow \mathbb{R}_{+}$by

$W(S, I, V)$

$$
\begin{aligned}
= & l\left[\frac{1}{2}\left(S-S^{*}\right)^{2}+a\left(I-I^{*}-I^{*} \log \frac{I}{I^{*}}\right)+\frac{1}{2} b\left(V-V^{*}\right)^{2}\right] \\
& +\frac{1}{2}\left[\left(S-S^{*}\right)+\left(I-I^{*}\right)+\left(V-V^{*}\right)\right]^{2}=l W_{1}+W_{2},
\end{aligned}
$$


where

$$
\begin{aligned}
W_{1}(S, I, V)= & \frac{1}{2}\left(S-S^{*}\right)^{2}+a\left(I-I^{*}-I^{*} \log \frac{I}{I^{*}}\right) \\
& +\frac{1}{2} b\left(V-V^{*}\right)^{2},
\end{aligned}
$$

$W_{2}(S, I, V)=\frac{1}{2}\left[\left(S-S^{*}\right)+\left(I-I^{*}\right)+\left(V-V^{*}\right)\right]^{2}$.

By Itô's formula, we compute

$$
d W_{1}=L W_{1} d t-\sigma S I\left(S-S^{*}\right) d B(t)+a \sigma S\left(I-I^{*}\right) d B(t),
$$

where

$$
\begin{aligned}
L W_{1}= & \left(S-S^{*}\right) d S+\frac{1}{2}(d S)^{2}+a\left(1-\frac{I^{*}}{I}\right) d I \\
& +b\left(V-V^{*}\right) d V+\frac{1}{2} b(d V)^{2} \\
= & \left(S-S^{*}\right)(\Lambda-\beta S I-(\mu+p) S+\varepsilon V)+\frac{1}{2} \sigma^{2} S^{2} I^{2} \\
& +a\left(I-I^{*}\right)[\beta S-(\mu+\gamma+\alpha)]+\frac{1}{2} \sigma^{2} S^{2} \\
& +b\left(V-V^{*}\right)[p S-(\mu+\varepsilon) V] \\
= & \left(S-S^{*}\right)\left[-\beta\left(S-S^{*}\right) I-\beta S^{*}\left(I-I^{*}\right)\right. \\
& \left.\quad-(\mu+p)\left(S-S^{*}\right)+\varepsilon\left(V-V^{*}\right)\right] \\
& +a \beta\left(S-S^{*}\right)\left(I-I^{*}\right) \\
& +b\left(V-V^{*}\right)\left[p\left(S-S^{*}\right)-(\mu+\varepsilon)\left(V-V^{*}\right)\right] \\
& +\frac{1}{2} \sigma^{2} S^{2} I^{2}+\frac{1}{2} \sigma^{2} S^{2} \\
= & -(\mu+p)\left(S-S^{*}\right)^{2}+(\varepsilon+b p)\left(S-S^{*}\right)\left(V-V^{*}\right) \\
& -b(\mu+\varepsilon)\left(V-V^{*}\right)^{2}-\beta\left(S-S^{*}\right)^{2} I \\
& +\beta\left(a-S^{*}\right)\left(S-S^{*}\right)\left(I-I^{*}\right)+\frac{1}{2} \sigma^{2} S^{2} I^{2}+\frac{1}{2} \sigma^{2} S^{2} .
\end{aligned}
$$

Here we choose $=S^{*}$, such that $\beta\left(a-S^{*}\right)=0$. Choose $b=\varepsilon / p$ and let $h=((\mu+\varepsilon)(\mu+p)+\varepsilon p) / 2 p(\mu+p)$; substituting this into (25) yields

$$
\begin{aligned}
L W_{1} \leqslant & -\left[(\mu+p)-\frac{\varepsilon}{h}\right]\left(S-S^{*}\right)^{2} \\
& -\left[\frac{\varepsilon}{p}(\mu+\varepsilon)-h \varepsilon\right]\left(V-V^{*}\right)^{2}+\frac{1}{2} \sigma^{2} S^{2} I^{2}+\frac{1}{2} \sigma^{2} S^{2}
\end{aligned}
$$

$$
\begin{aligned}
\leqslant & -\frac{\mu(\mu+\varepsilon+p)(\mu+p)}{(\mu+\varepsilon)(\mu+p)+\varepsilon p}\left(S-S^{*}\right)^{2} \\
& -\frac{\mu \varepsilon(\mu+\varepsilon+p)}{2 p(\mu+p)}\left(V-V^{*}\right)^{2} \\
& +\frac{1}{2} \sigma^{2}\left[\left(\frac{\Lambda}{\mu}\right)^{4}+\left(\frac{\Lambda}{\mu}\right)^{2}\right] .
\end{aligned}
$$

Similarly, applying Itô's formula to $W_{2}(S, I, V)$, we get

$$
\begin{aligned}
L W_{2}= & {\left[\left(S-S^{*}\right)+\left(I-I^{*}\right)+\left(V-V^{*}\right)\right](d S+d I+d V) } \\
& +\frac{1}{2}((d S)+(d I)+(d V))^{2} \\
= & {\left[\left(S-S^{*}\right)+\left(I-I^{*}\right)+\left(V-V^{*}\right)\right] } \\
& \times\left[-\mu\left(S-S^{*}\right)-(\mu+\gamma+\alpha)\left(I-I^{*}\right)-\mu\left(V-V^{*}\right)\right] \\
= & -\mu\left(S-S^{*}\right)^{2}-(\mu+\gamma+\alpha)\left(I-I^{*}\right)^{2}-\mu\left(V-V^{*}\right)^{2} \\
& -(2 \mu+\gamma+\alpha)\left(S-S^{*}\right)\left(I-I^{*}\right) \\
& -(2 \mu+\gamma+\alpha)\left(I-I^{*}\right)\left(V-V^{*}\right) \\
& -2 \mu\left(S-S^{*}\right)\left(V-V^{*}\right) \\
\leqslant & (2 \mu+\gamma+\alpha)\left(S-S^{*}\right)^{2}-\frac{\gamma+\alpha}{2}\left(I-I^{*}\right)^{2} \\
& +(2 \mu+\gamma+\alpha)\left(V-V^{*}\right)^{2} .
\end{aligned}
$$

Therefore, we can obtain

$$
\begin{aligned}
L W(S, I, V)= & l L W_{1}+L W_{2} \\
\leqslant & -\left[l \frac{\mu(\mu+\varepsilon+p)(\mu+p)}{(\mu+\varepsilon)(\mu+p)+\varepsilon p}-(2 \mu+\gamma+\alpha)\right] \\
& \times\left(S-S^{*}\right)^{2}-\frac{\gamma+\alpha}{2}\left(I-I^{*}\right) 2 \\
& -\left[l \frac{\mu \varepsilon(\mu+\varepsilon+p)}{2 p(\mu+p)}-(2 \mu+\gamma+\alpha)\right] \\
& \times\left(V-V^{*}\right)^{2}+\frac{1}{2} \sigma^{2}\left[\left(\frac{\Lambda}{\mu}\right)^{4}+\left(\frac{\Lambda}{\mu}\right)^{2}\right] \\
:= & F(t) .
\end{aligned}
$$

Let us choose

$$
\begin{gathered}
l>\max \left\{\frac{(\mu+\varepsilon)(\mu+p)+\varepsilon p}{\mu(\mu+\varepsilon+p)(\mu+p)}(2 \mu+\gamma+\alpha),\right. \\
\left.\frac{2 p(\mu+p)(2 \mu+\gamma+\alpha)}{\mu \varepsilon(\mu+\varepsilon+p)}\right\},
\end{gathered}
$$


such that

$$
\begin{gathered}
\frac{l \mu(\mu+\varepsilon+p)(\mu+p)}{(\mu+\varepsilon)(\mu+p)+\varepsilon p}-(2 \mu+\gamma+\alpha)>0, \\
\frac{l \mu \varepsilon(\mu+\varepsilon+p)}{2 p(\mu+p)(2 \mu+\gamma+\alpha)}-(2 \mu+\gamma+\alpha)>0 .
\end{gathered}
$$

Therefore, we have

$$
d W \leqslant F(t) d t+\sigma S\left[S^{*}\left(I-I^{*}\right)-\left(S-S^{*}\right) I\right] d B(t) .
$$

Integrating it from 0 to $t$ yields

$$
\begin{aligned}
W(t)-W(0) \leqslant & \int_{0}^{t} F(s) d s \\
& +\int_{0}^{t} \sigma S\left[S^{*}\left(I-I^{*}\right)-\left(S-S^{*}\right) I\right] d B(s) .
\end{aligned}
$$

Let

$$
M(t)=\int_{0}^{t} \sigma S\left[S^{*}\left(I-I^{*}\right)-\left(S-S^{*}\right) I\right] d B(s),
$$

which is a continuous local martingale and $M(0)=0$. Moreover,

$$
\langle M, M\rangle_{t}=\int_{0}^{t} \sigma^{2} S^{2}\left[S^{*}\left(I-I^{*}\right)-\left(S-S^{*}\right) I\right]^{2} d t
$$

and by (9) then

$$
\begin{aligned}
\limsup _{t \rightarrow \infty} \frac{1}{t}\langle M, M\rangle_{t} & \leqslant \limsup _{t \rightarrow \infty} \sigma^{2} S^{2}\left[S^{*}\left(I-I^{*}\right)-\left(S-S^{*}\right) I\right]^{2} \\
& \leqslant 16 \sigma^{2}\left(\frac{\Lambda}{\mu}\right)^{6}<\infty \text { a.s. }
\end{aligned}
$$

This together with Lemma 4 implies

$$
\begin{aligned}
\limsup _{t \rightarrow \infty} \frac{M(t)}{t} \\
\quad=\frac{\int_{0}^{t} \sigma S\left[S^{*}\left(I-I^{*}\right)-\left(S-S^{*}\right) I\right] d B(s)}{t}=0 \text { a.s. }
\end{aligned}
$$

Consequently, combining (36), from (32) we get

$$
\begin{gathered}
\limsup _{t \rightarrow \infty} \frac{1}{t} \int_{0}^{t}\left\{\left[l \frac{\mu(\mu+\varepsilon+p)(\mu+p)}{(\mu+\varepsilon)(\mu+p)+\varepsilon p}-(2 \mu+\gamma+\alpha)\right]\right. \\
\times\left(S(r)-S^{*}\right)^{2}+\frac{\gamma+\alpha}{2}\left(I(r)-I^{*}\right)^{2} \\
+\left[l \frac{\mu \varepsilon(\mu+\varepsilon+p)}{2 p(\mu+p)}-(2 \mu+\gamma+\alpha)\right] \\
\left.\quad \times\left(V(r)-V^{*}\right)^{2}\right\} d r \\
\leqslant \frac{1}{4} \sigma^{2}\left(\frac{\Lambda}{\mu}\right)^{2}\left(1+\left(\frac{\Lambda}{\mu}\right)^{2}\right) \text { a.s. }
\end{gathered}
$$

Thus, the proof of Theorem 6 is completed.
Remark 7. For system (2), if $\sigma=0$, from Theorem 6, then

$$
\begin{aligned}
\limsup _{t \rightarrow \infty} \frac{1}{t} \int_{0}^{t}\{[l & {\left[\frac{\mu(\mu+\varepsilon+p)(\mu+p)}{(\mu+\varepsilon)(\mu+p)+\varepsilon p}-(2 \mu+\gamma+\alpha)\right] } \\
& \times\left(S(r)-S^{*}\right)^{2}+\frac{\gamma+\alpha}{2}\left(I(r)-I^{*}\right)^{2} \\
& +\left[l \frac{\mu \varepsilon(\mu+\varepsilon+p)}{2 p(\mu+p)}-(2 \mu+\gamma+\alpha)\right] \\
& \left.\times\left(V(r)-V^{*}\right)^{2}\right\} d r=0,
\end{aligned}
$$

which implies

$$
\lim _{t \rightarrow \infty} S(t)=S^{*}, \quad \lim _{t \rightarrow \infty} I(t)=I^{*}, \quad \lim _{t \rightarrow \infty} V(t)=V^{*}
$$

which also shows that the solution tends to $E^{*}$ if $R_{0}>1$. Moreover, the result of Theorem 6 tells us the difference between $(S(t), I(t), V(t))$ and $E^{*}$ is proportional to the intensity of the white noise in time average. The smaller the white noise is, the closer the solution getting to $E^{*}$ is.

\section{Conflict of Interests}

The authors declare that there is no conflict of interests regarding the publication of this paper.

\section{Acknowledgments}

The work was supported by Program for Changjiang Scholars and Innovative Research Team in University, NSFC of China (no. 11371085), the Ph.D. Programs Foundation of Ministry of China (no. 200918), and the Scientific and Technological Research Project of Jilin Province's Education Department (no. 2014526).

\section{References}

[1] W. O. Kermack and A. G. McKendrick, "Contributions to the mathematical theory of epidemics-I. 1927," Bulletin of Mathematical Biology, vol. 53, no. 1-2, pp. 33-55, 1991.

[2] J. Li and Z. Ma, "Qualitative analyses of SIS epidemic model with vaccination and varying total population size," Mathematical and Computer Modelling, vol. 35, no. 11-12, pp. 1235-1243, 2002.

[3] J. Arino, C. C. Mccluskey, and P. V. van den Driessche, "Global results for an epidemic model with vaccination that exhibits backward bifurcation," SIAM Journal on Applied Mathematics, vol. 64, no. 1, pp. 260-276, 2003.

[4] A. D’Onofrio, "Mixed pulse vaccination strategy in epidemic model with realistically distributed infectious and latent times," Applied Mathematics and Computation, vol. 151, no. 1, pp. 181187, 2004.

[5] X. Meng, L. Chen, and H. Cheng, "Two profitless delays for the SEIRS epidemic disease model with nonlinear incidence and pulse vaccination," Applied Mathematics and Computation, vol. 186, no. 1, pp. 516-529, 2007. 
[6] G. Zeng, L. Chen, and L. Sun, "Complexity of an SIR epidemic dynamics model with impulsive vaccination control," Chaos, Solitons and Fractals, vol. 26, no. 2, pp. 495-505, 2005.

[7] L. Imhof and S. Walcher, "Exclusion and persistence in deterministic and stochastic chemostat models," Journal of Differential Equations, vol. 217, no. 1, pp. 26-53, 2005.

[8] D. Q. Jiang and N. Z. Shi, "A note on nonautonomous logistic equation with random perturbation," Journal of Mathematical Analysis and Applications, vol. 303, no. 1, pp. 164-172, 2005.

[9] D. Q. Jiang, N. Z. Shi, and X. Y. Li, "Global stability and stochastic permanence of a non-autonomous logistic equation with random perturbation," Journal of Mathematical Analysis and Applications, vol. 340, no. 1, pp. 588-597, 2008.

[10] Q. Lu, "Stability of SIRS system with random perturbations," Physica A: Statistical Mechanics and Its Applications, vol. 388, no. 18, pp. 3677-3686, 2009.

[11] R. R. Sarkar, "A stochastic model for autotroph-herbivore system with nutrient reclycing," Ecological Modelling, vol. 178, no. 3-4, pp. 429-440, 2004.

[12] E. Tornatore, S. M. Buccellato, and P. Vetro, "Stability of a stochastic SIR system," Physica A: Statistical Mechanics and Its Applications, vol. 354, no. 1-4, pp. 111-126, 2005.

[13] J. J. Yu, D. Q. Jiang, and N. Z. Shi, "Global stability of two-group SIR model with random perturbation," Journal of Mathematical Analysis and Applications, vol. 360, no. 1, pp. 235-244, 2009.

[14] Y. Zhao, D. Jiang, and D. O'Regan, "The extinction and persistence of the stochastic SIS epidemic model with vaccination," Physica A: Statistical Mechanics and Its Applications, vol. 392, no. 20, pp. 4916-4927, 2013.

[15] Y. Zhao and D. Jiang, "Dynamics of stochastically perturbed SIS epidemic model with vaccination," Abstract and Applied Analysis, vol. 2013, Article ID 517439, 12 pages, 2013.

[16] Y. Zhao and D. Jiang, "The threshold of a stochastic SIRS epidemic model with saturated incidence," Applied Mathematics Letters, 2013.

[17] X. Mao, G. Marion, and E. Renshaw, "Environmental Brownian noise suppresses explosions in population dynamics," Stochastic Processes and Their Applications, vol. 97, no. 1, pp. 95-110, 2002.

[18] X. Mao, Stochastic Differential Equations and Applications, Ellis Horwood, Chichester, UK, 1997. 


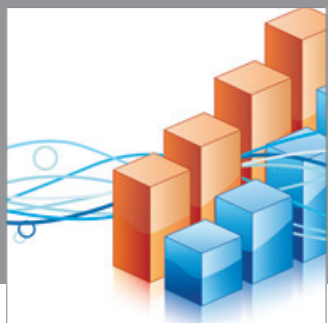

Advances in

Operations Research

mansans

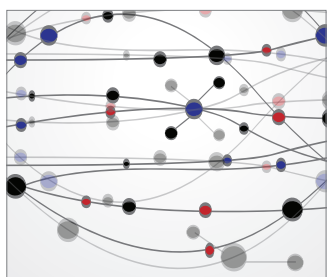

The Scientific World Journal
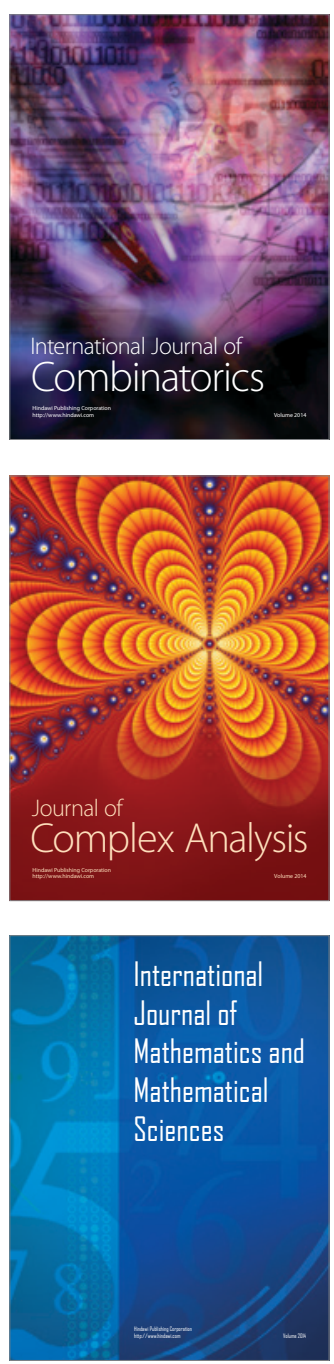
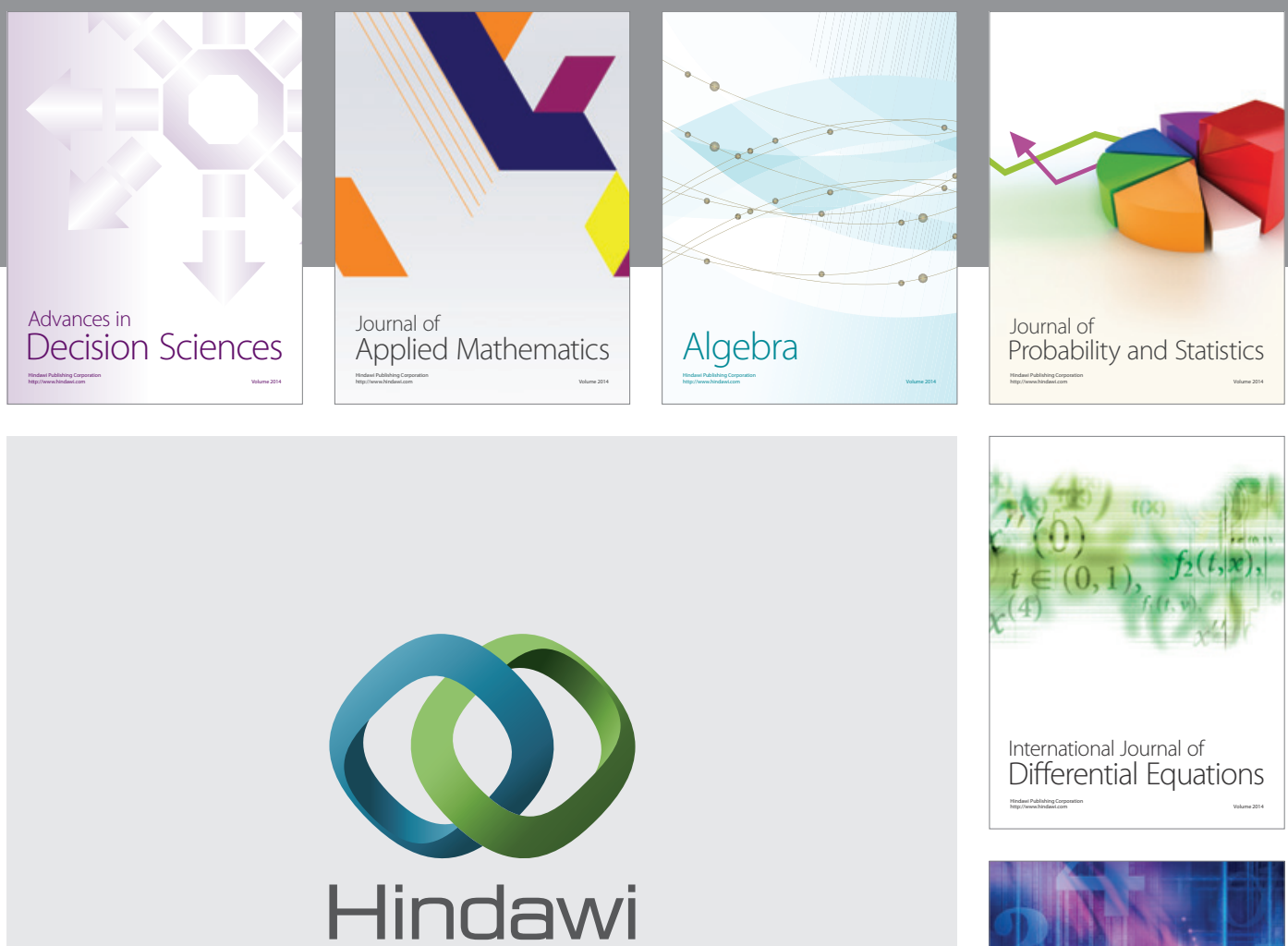

Submit your manuscripts at http://www.hindawi.com
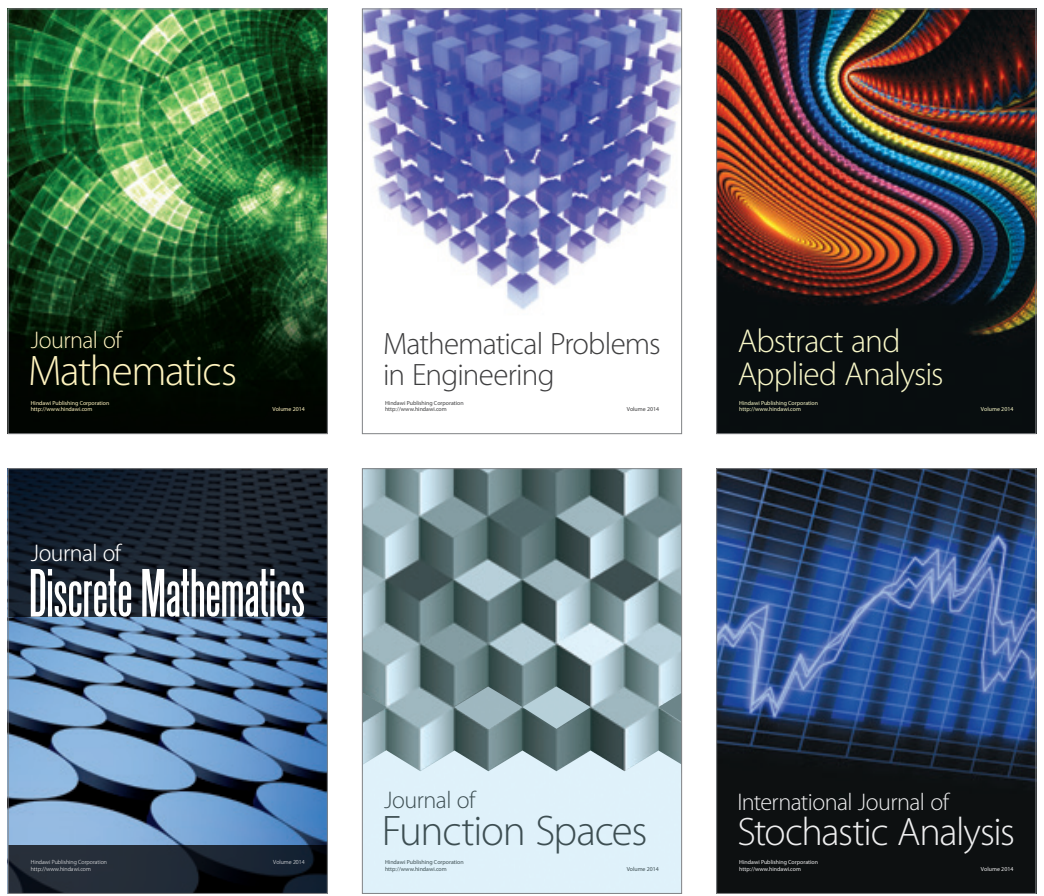

Journal of

Function Spaces

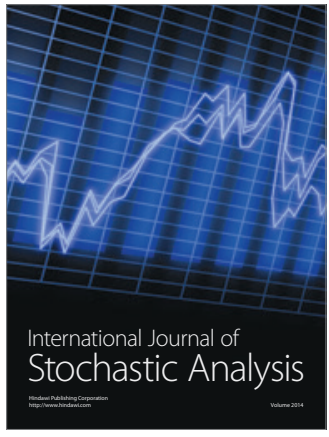

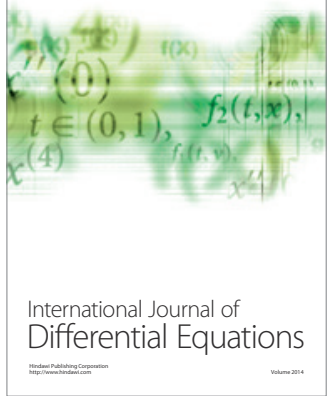
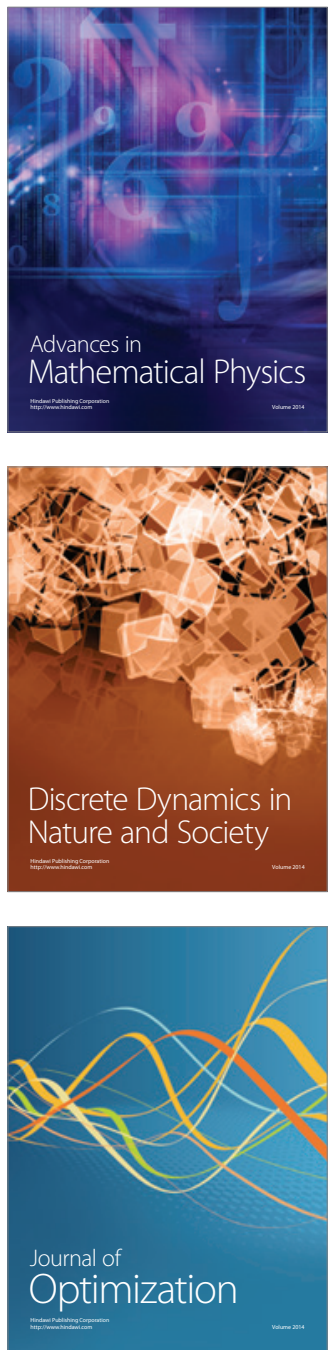